\title{
DER IMAGINÄRE BÜHNENRAUM IN SENECAS HERCULES FURENS
}

\section{Alexander Kirichenko}

Humboldt-Universität zu Berlin, Philosophische Fakultät II, Institut für Klassische Philologie, Unter den Linden 6, D-10099 Berlin, kirichen@uni-trier.de

The goal of this article is to analyze the construction of space in Seneca's Hercules Furens. It argues that the tragedy establishes a multi-tiered structural analogy among three conceptual domains -1 ) the world of supernatural myth, which cannot be 'shown' on stage and which, for that reason, is described in rhetorically overblown ekphrastic passages, 2) the visually perceptible world of the stage action, and 3) the abstract world of 'human life. These three conceptual domains form an imaginary hybrid space within which philosophically relevant thought is not so much formulated as staged, thereby producing an emotionally powerful - and potentially transformative - effect on the recipient. In addition to offering a new interpretation of Hercules Furens, this article thus sheds some important light on the vexed issue of the philosophical intent of Senecan tragedy in general.

Keywords: Seneca, Hercules Furens, tragedy, philosophy

Die Forschung zu den Tragödien Senecas ist seit Jahrzehnten von zwei Oppositionen geprägt, die dafür sorgen, dass viele interpretationsrelevante Probleme als kompromisslose „entweder/oder Fragen“ formuliert werden. Zum einen versucht man vergeblich, eine Einigkeit in der Frage zu erzielen, ob es sich bei Senecas Tragödien um rein literarische Darstellungen oder um rein philosophische Lehrstücke handelt. ${ }^{1}$ Zum anderen bemüht man sich unermüdlich darum, den Status der senecanischen Tragödien entweder als zur Aufführung bestimmte Theaterstücke oder als nur für private Lektüre geeignete literarische Texte mit ähnlicher Eindeutigkeit zu bestimmen. ${ }^{2}$ Da es bekanntlich keine direk-

${ }^{1}$ Die Ähnlichkeiten zwischen ausgewählten tragischen und philosophischen Passagen der senecanischen Werke verleiten einige Forscher dazu, dass sie auch in den Tragödien ausschließlich Moralpredigten sehen, die ihr Ziel durch abschreckende Darstellungen der für das philosophisch gesinnte Publikum mit allen Mitteln zu vermeidenden Verhaltensmuster erreichen. Z. B. Regenbogen 1927/1928, Marti 1945, Pratt 1983, Lefèvre 1985 und, viel moderater und nuancierter, Rosenmeyer 1989 und Staley 2010. Zu Seneca tragicus als einem Antipoden des Seneca philosophus siehe z. B. Dingel 1974. Zur radikalen Trennung zwischen Senecas Tragödiendichtung und seinen philosophischen Schriften siehe z. B. Schiesaro 2003; Littlewood 2004. Alessandro Schiesaro betrachtet die Möglichkeit einer von einem stoischen Philosophen gedichteten Tragödie gar als einen unlösbaren logischen Widerspruch, Schiesaro 2003, 253: „What we ultimately face is the impossibility of Stoic tragedy. For sapientes will have no interest in it, and proficientes are as likely to be deceived by it as they are to draw useful precepts. "Vgl. Chaumartin 2014; Winter 2014, 16-20; Trinacty 2015, 36-38.

2 Die Anhänger der These, Senecas Tragödien seien in der Antike aufgeführt worden, berufen sich auf die Szenen von außerordentlicher dramatischer Intensität, die es fast in jedem senecanischen Stück gibt, auf die metatheatralische Selbstreflexivität der tragischen Charaktere, sowie auf die durch die moderne Praxis bestätigte grundsätzliche Aufführbarkeit der Tragödien: z. B. Tarrant 1978, Sutton 1986 und die meisten Beiträge in Harrison 2000. Diejenigen hingegen, die in Senecas Tragödien Deklamations- bzw. Lesedramen sehen, lenken ihr Augenmerk erwartungsgemäß vor allem auf Illusionsbrüche, die auf einer den stringent mimetischen Erfordernissen entsprechenden tragischen Bühne undenkbar wären, sowie auf statische deskriptive Passagen, die in einem richtigen Theaterstück wie ein unzumutbarer Fremdkörper erscheinen würden. Dazu siehe vor allem Zwierlein 1966; Zwierleins Argumente für Senecas Tragödien als Rezitationsdramen wurden neulich von Christoph Kugelmeier 2007 überprüft und weitgehend bestätigt. Zu Senecas Tragödien als Lesedramen s. Fantham 1982, 34-49. Kohn 2014 analysiert die Dramaturgie der senecanischen Tragödien, ohne sich in der Aufführungsdebatte eindeutig zu bekennen. Vgl. Trinacty 2015, 32-36.

(c) St. Petersburg State University, 2016 
ten antiken Zeugnisse gibt, die eine dieser Theorien unwiderlegbar beweisen könnten, ${ }^{3}$ verwandeln sich die auf Grundlage solcher binären Logik geführten Forschungsdebatten notgedrungen in reine Glaubensbekenntnisse, bei denen man zur Bekräftigung seiner eigenen Überzeugung das eine Element der Dichotomie hervorzuheben und das andere als zweitrangig und somit als weniger beachtenswert zu behandeln tendiert. Das Ziel dieses Aufsatzes besteht darin, eine Alternative zur üblichen binären Logik der Seneca-Forschung anzubieten. Am Beispiel einer Tragödie (des Hercules Furens) soll gezeigt werden, dass die Wahrnehmung der senecanischen Stücke in erster Linie durch die Konstruktion ihres imaginären Bühnenraums bestimmt wird. Innerhalb dieser Konstruktion kann man zwischen drei ontologischen Ebenen unterscheiden: 1) der Welt des übernatürlichen Mythos, der sich auf der Bühne mimetisch nicht darstellen lassen kann und darum in der Regel in rein deskriptiven - ekphrastischen - Passagen beschrieben wird; 2) der Bühnenwelt, in der nur in der empirischen Realität denkbare Handlungen stattfinden; und 3) der Welt diesseits der Bühne, in der die Rezipienten der Tragödie (ob es sich dabei um Zuschauer oder Leser handelt, ist für die Konstruktion des imaginären Bühnenraums von geringer Relevanz) durch die Bühnenhandlung aufgefordert werden, ihre eigene Selbstwahrnehmung zu ändern. Diese drei Räume - der Bereich des fantastischen Mythos, die Bühne und das Ich des Rezipienten - werden aufeinander projiziert und formen als Folge das, was man in der modernen Kognitionswissenschaft als „blended mental space“ bezeichnen würde, ${ }^{4}$ - einen hybriden imaginären Raum, der etwas zu konzeptualisieren erlaubt, was sich ohne diese doppelte Fiktion gar nicht (oder zumindest nicht mit der gleichen Überzeugungskraft) zeigen ließe. Die binären Oppositionen, die der SenecaForschung traditionell zugrunde liegen, sollen somit einer komplexen konzeptuellen Vermischung weichen, in der die private Lektüre der Tragödien notgedrungen zu einer zwar imaginierten, doch auch empirisch realisierbaren Bühnenaufführung wird und in der die Stücke dank, und nicht trotz, ihrer mimetischen Konstruiertheit als genuin philosophische Dramen betrachtet werden können.

Im Prolog zum Hercules Furens werden sowohl die zentralen Themen der Tragödie als auch die höchst schematische Beschaffenheit ihrer Raumkonstruktion und Handlung angedeutet. Die Prologsprecherin Juno beklagt sich zunächst über die zunehmend besorgniserregende Allmächtigkeit des Hercules: ${ }^{5}$ Nachdem er alle Monster der Erde besiegt und nun mit der Vollendung seiner größten Heldentat (der Bezwingung der Un-

${ }^{3}$ Es gibt keinerlei zeitgenössische Zeugnisse, die Senecas Tragödien direkt erwähnen: Sogar Senecas eigene zahlreiche Prosaschriften (abgesehen von einem möglichen Zitat in der Apocolocyntosis: vgl. Sen. Apoc. 12, 1-2: fundite fletus, edite planctus, / resonet tristi clamore forum; Sen. Tr. 131: fundite fletus; Sen. HF 1108: resonet maesto clamore chaos; dazu siehe Nisbet 1990, 95-97) enthalten keinen einzigen direkten Hinweis auf seine Theaterstücke! Selbst die traditionelle Zuordnung der senecanischen Tragödien zur „neronischen“ Literatur ist keineswegs gesichert. Vgl. Tarrant 1976, 7: „What should be clear, however, is that the fashionable interpretation of these works as ,Neronian' has no secure basis in fact; they could with equal justification be regarded as Claudian, Gaian, or even Tiberian."

${ }^{4}$ Zur „conceptual integration“ oder „conceptual blending“ als der Grundlage des menschlichen Denkens siehe Turner 1996 und Fauconier - Turner 2002.

5 Zur Juno-Gestalt im Prolog zu Senecas HF siehe Shelton 1978, 17-25; Lawall 1983, 6-10; Guastella 2001, 20-22; Schiesaro 2003, 183-186; Li Causi 2006, 120-127; Wiener 2006, 84-90; Fischer 2008, 57-91; Trinacty 2014, 130-138. 
terwelt) eine bisher als absolut geltende Grenze überschritten habe, bestehe die durchaus begründete Befürchtung, er könne nun auch den Himmel erobern wollen und dadurch die gesamte bestehende Weltordnung durcheinander bringen. ${ }^{6}$ Wir sehen sofort, dass Hercules in erster Linie als ein gefährliches Paradoxon dargestellt wird, das die räumliche Ordnung - die dreiteilige hierarchische Struktur des Universums - in den Zustand des ursprünglichen Chaos zurückstößt und somit die scheinbar entgegengesetzten kognitiven Kategorien miteinander vermischt. Dieses monströse Geschöpf muss nun beseitigt werden, was nach der schematischen Logik des Prologs nur durch Hercules selbst geschehen kann (es gibt ja schließlich nach der Vollendung seiner Heldentaten niemanden mehr auf der Welt, der ihn herausfordern könnte). ${ }^{7}$ Die Unantastbarkeit der räumlichen Struktur der Welt kann folglich nur dadurch wiederherstellt werden, dass Hercules die letzte Konsequenz aus der sein Wesen bestimmenden Vermischung zwischen dem größten Helden und dem größten Monster zieht, wodurch sein Selbstmord zu einer Überbietung einer herculeischen Heldentat werden müsste.

Die Konfrontation mit dem inneren Chaos kann aber für Hercules nur als Folge seiner Konfrontation mit dem räumlichen Chaos eintreten - einer Vermischung zwischen der empirischen Realität und der Welt des übernatürlichen Mythos, die er besiegt hat. Es ist höchst bezeichnend, dass Juno die Katabasis des Hercules als eine frevelhafte Offenbarung der Geheimnisse der Unterwelt auffasst - als das Sichtbarmachen von etwas, was für immer unsichtbar bleiben sollte. ${ }^{8}$ Die darauf folgende detaillierte Beschreibung von Gestalten der Unterwelt, die Hercules zur Selbstzerstörung bewegen sollen, ${ }^{9}$ zeigt, dass diese einmalige Offenbarung zu einer verhängnisvollen kognitiven Verwirrung führen muss, was Juno mit unmissverständlicher Klarheit betont, als sie Hercules verspricht, dass er erst hier (hic) $)^{10}$ die echte Unterwelt erleben wird - hier auf der Bühne, wo man sie am wenigsten erwartet. Die gesamte darauf folgende Handlung lässt sich als eine permanente Oszillierung zwischen einer Aufspaltung und einer Vermischung von verschiedenen räumlichen und konzeptuellen Bereichen verstehen.

Am Ende des Prologs des Hercules Furens führt Juno den gerade einsetzenden Tagesanbruch als Hauptgrund dafür an, dass sie mit der Ausführung ihres Plans möglichst schnell beginnen muss. ${ }^{11}$ Dieser konkrete Naturvorgang dient dem Chor als Ausgangs-

${ }^{6}$ Sen. HF 64-68: caelo timendum est, regna ne summa occupet / qui vicit ima: sceptra praeripiet patri. / nec astra lenta veniet ut Bacchus via: / iter ruina quaeret et vaculo volet / regnare mundo. Dazu siehe Schmitz 1993, 116-118; Littlewood 2004, 107-127; Kirichenko 2013, 17-18.

7 Sen. HF 84-85: quaeris Alcidae parem? / nemo est nisi ipse: bella iam secum gerat. Zur Gestalt des senecanischen Hercules und zu ihren Verbindungen mit den Hercules-Darstellungen in der griechischen und römischen Literatur siehe Fitch 1987, 15-20; Billerbeck 1999, 1-29.

${ }^{8}$ Sen. HF 51-60: en retegit Styga! / patefacta ab imis manibus retro via est / et sacra dirae mortis in aperto iacent. / at ille, rupto carcere umbrarum ferox, / de me triumphat et superbifica manu / atrum per urbes ducit Argolicas canem. I viso labantem Cerbero vidi diem / pavidumque Solem; me quoque invasit tremor, / et terna monstri colla devicti intuens / timui imperasse.

9 Sen. HF 86-88: adsint ab imo Tartari fundo excitae / Eumenides, ignem flammeae spargant comae, I viperea saevae verbera incutiant manus. Vgl. Solimano 1998, 241-242. Besonders bezeichnend ist dabei, dass Seneca die euripideische Lyssa durch Gestalten ersetzt, deren Schilderung der vergilischen Darstellung der Unterwelt Einiges zu verdanken hat. Siehe auch Staley 2010, 98-100.

${ }_{10}$ Sen. HF 89-91: i nunc, superbe, caelitum sedes pete, / humana temne. iam Styga et manes, ferox, / fugisse credis? hic tibi ostendam inferos. Vgl. Wessels 2014, 82-85.

${ }^{11}$ Sen. HF 123-124: movenda iam sunt bella: clarescit dies / ortuque Titan lucidus croceo subit. Zur Bedeutung dieser Zeitangabe siehe Heil 2013, 76-82. 
punkt für seinen ersten Auftritt. ${ }^{12}$ Wenn man alle Chorlieder im Hercules Furens zusammen betrachtet, könnte man sagen, dass ihre Funktion, wie auch in anderen senecanischen Tragödien, ${ }^{13}$ in erster Linie darin besteht, einerseits den Status des tragischen Mythos als einer bloßen poetischen Fiktion und andererseits die Notwendigkeit dieser Fiktion für die Darstellung der sonst kaum darstellbaren Aspekte der menschlichen Existenz zu betonen. Dieser Prozess der Reflexion über die Natur des Mythischen beginnt gleich im ersten Chorlied. Der Chor beschreibt zunächst den Tagesanbruch in der Begrifflichkeit des tragischen Mythos - als eine Art Projektionsfläche für solche mythologischen Gestalten wie Callisto, Agave und Philomela. ${ }^{14}$ Im weiteren Verlauf dieser Beschreibung entfernt sich der Chor sukzessive von dieser mythologischen Perspektive und zeigt dadurch, dass er sich gewissermaßen außerhalb der mythologischen Bühnenwelt befindet. Der Kontrast zur künstlichen - mythologischen, tragischen - Welt ist dabei frappierend: Anstelle der verwandelten Königstöchter und der von ihren Müttern zerstückelten oder ihrem eigenen Vater zum Verzehr dargebotenen Kinder tritt die noble Arbeit eines Hirten, eines Seemanns und eines Fischers, die allesamt aufwachen, um ihren Alltag zu beginnen. ${ }^{15} \mathrm{Im}$ nächsten Schritt wird das einfache, mit dem grenzenlosen Glück gleichgesetzte Arbeitsleben mit der korrupten Welt von nach Profit gierigen Großstädtern kontrastiert. ${ }^{16}$ Dieser doppelte Kontrast lässt also auch die mythologische Welt als die mit dem wahren Glück unvereinbare Domäne der Äußerlichkeiten erscheinen. ${ }^{17}$ Hercules' Katabasis wird anschließend als Paradebeispiel eines solchen Eintauchens in die illusorische Welt des Mythos und gleichzeitig als eine vollkommen sinnlose Grenzüberschreitung aufgefasst (wofür diese Eile, wenn man sowieso früher oder später sterben muss?). ${ }^{18}$ Die moralphilosophische Plattitüde, mit der der Chor seinen Auftritt beendet (das einfache Leben im Verborgenen sei besser als die illusorischen Vorzügen der mythologischen Heldenhaftigkeit), ${ }^{19}$ verfestigt endgültig die provisorische Trennung zwischen der gesamten mythologischen Bühnenhandlung und der entmythologisierten Außenperspektive des Chors.

Nach dem ersten Chorlied gelten die drei räumlichen Komponenten des Dramas der übernatürliche, der szenische und der außerszenische Raum - als völlig etabliert, und die Handlung besteht hauptsächlich in einer gegenseitigen Projizierung dieser drei Räume aufeinander. Im zweiten Akt wird die Gültigkeit der übernatürlichen Welt, im Einklang mit dem ersten Chorlied, dadurch in Frage gestellt, dass Lycus, der während Hecules' Abwesenheit dessen Thron usurpiert hat, sowohl an der göttlichen Abstammung des

12 Zu diesem Chorlied siehe Mader 1990; Davis 1993, 126-136; Kirichenko 2013, 267-270.

13 Zu Senecas Chorpassagen im Allgemeinen siehe Kirichenko 2013, 249-279.

14 Sen. HF 128-140: cogit nitidum Phosphoros agmen, / signum celsi glaciale poli / septem stellis Arcados ursae / lucem verso temone vocat. / iam caeruleis evectus aquis / Titan summa prospicit Oeta; / iam Cadmeis incluta Bacchis / aspersa die dumeta rubent / Phoebique fugit reditura soror. / Pendet summo stridula ramo / pinnasque novo tradere soli / gestit querulos inter nidos / Thracia paelex.

15 Sen. HF 139-158. Für Thomas Gärtner 2003, 3-4, ist das erster Chorlied des HF deswegen das anschaulichste Beispiel, das seine These belegen soll, die primäre Funktion des Chors in Senecas Tragödien bestehe ausschließlich darin, die Maxime, „,besser, dem gemeinen Volk anzugehören“ (so lautet nämlich der Titel seines Aufsatzes), zu illustrieren.

16 Sen. HF 159-161: haec innocuae quibus est vitae / tranquilla quies / et laeta suo parvoque domus.

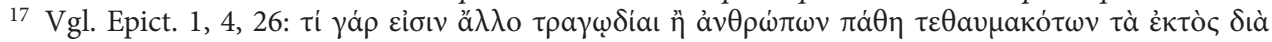

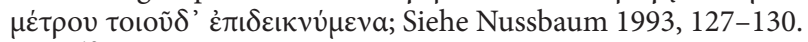

18 Sen. HF 186-191: nimium, Alcide, pectore forti / properas maestos visere manes: / certo veniunt tempore Parcae, / nulli iusso cessare licet, / nulli scriptum proferre diem: / recipit populos urna citatos.

19 Sen. HF 198-201: venit ad pigros cana senectus, / humilique loco sed certa sedet / sordida parvae fortuna domus: / alte virtus animosa cadit. Siehe dazu Degl'Innocenti Pierini 1996. 
Hercules als auch an der Möglichkeit dessen Rückkehr aus der Unterwelt zweifelt. ${ }^{20}$ Das zweite Chorlied setzt anschließend die Transformation des Übernatürlichen in eine visuell beeindruckende Metapher für die allzu menschliche Bühnenhandlung. An der Oberfläche besteht das rhetorische telos dieses Chorlieds darin, Hercules' heroische Katabasis mit Orpheus' „poetischer" Katabasis zu parallelisieren und dadurch die bevorstehende Rückkehr des Hercules aus der Unterwelt vorwegzunehmen: Denn wenn es jemandem gelungen sei, der nur ein Dichter war, so die Logik dieser Passage, werde es mit Sicherheit auch dem größten aller Helden gelingen. Besonders wichtig ist jedoch, dass die detaillierte Erzählung von Orpheus' Erfolg bei dem Versuch, Euridike aus der Unterwelt zu befreien, mit einer scheinbar beiläufigen Erwähnung seines tragischen Fehlers endet, der zum Verlust seiner Ehefrau führte. ${ }^{21}$ Dadurch wird einerseits unsere Aufmerksamkeit auf die illusorische Natur des Sieges des Orpheus gelenkt und andererseits das Ende der Tragödie vorweggenommen, denn genauso wie Orpheus wird auch Hercules am Ende seine Frau durch seine eigene Verirrung verlieren. Noch aussagekräftiger ist die direkte Parallelisierung zwischen der heroischen Kraft des Hercules und der poetischen Kraft des Orpheus. Hercules wird laut dem Chor die Unterwelt auf die gleiche Weise besiegen, wie es Orpheus schon einmal getan hat, ${ }^{22}$ - nämlich gar nicht, da die echte Hölle, wie Juno bereits im Prolog betonte, für ihn auch erst nach seinem vermeintlichen Sieg anfängt. Die von Hercules vollbrachte Heldentat wird somit indirekt mit Orpheus' Dichtung, das Heroische mit der Literatur gleichgesetzt.

Der dritte Akt bringt den Rezipienten der im Prolog bereits angedeuteten Transformation des Bühnenraums in eine Version der Unterwelt einen Schritt näher. Als Hercules nach seiner Rückkehr wieder die Bühne verlässt, um, gemäß seiner heroischen Natur, seinen Widersacher Lycus sofort zu töten, nützt Amphitryon die daraus entstehende Handlungspause, um Theseus, der Hercules auf seiner Unterweltreise begleitet hatte, ausführlich nach den Einzelheiten der Katabasis zu befragen. Die darauf folgende fast hundertsiebzig Verse lange ekphrastische Passage (Sen. HF 658-829), die auf zahlreichen aus der früheren Literatur - von Homer über die platonischen Mythen bis hin zu Vergil - bekannten Klischees basiert, ${ }^{23}$ bewirkt eine Verstärkung der im Prolog angedeuteten Sichtbarwerdung des Unsichtbaren. Besonders wichtig für die Konstruktion des Bühnenraums ist jedoch, dass die Beschreibung des Rückweges aus der Unterwelt nicht durch die menschlichen Augen des Theseus, oder des Hercules, fokalisiert wird, sondern durch die Augen des Cerberus, den Hercules aus der Unterwelt entführt. Die Tatsache, dass wir die vertraute Ansicht der empirischen Welt aus dem Blickwinkel eines infernalischen Ungeheuers sehen, konstituiert einen höchst ausgeklügelten Kunstgriff: Für Cerberus stellt die vom Sonnenlicht überflutete Welt eine genauso schwer zu bewältigende visuelle Herausforderung dar wie die Finsternis des Hades für Hercules und Theseus. ${ }^{24}$ Die Tatsache,

${ }^{20}$ Sen. HF 397-413, 432, 465-471. Zur Lycus-Gestalt bei Seneca siehe Shelton 1978, 32-37; Kirichenko 2013, 20-21.

${ }^{21}$ Sen. HF 588-589: odit verus amor nec patitur moras: / munus dum properat cernere, perdidit

22 Sen. HF 590-591: quae vinci potuit regia carmine, / haec vinci poterit regia viribus. Vgl. Fitch 1987, 253; Heil 2013, 103; Kirichenko 2013, 263-264; Trinacty 2014, 157-158.

${ }^{23} \mathrm{Zu}$ dieser Passage im Allgemeinen siehe Shelton 1978, 50-57; Aygon 2004, 197-207; Kirichenko 2013, 24-27. Zu den literarischen Einflüssen siehe Fitch 1987, 293; Trinacty 2014, 36-37. Vgl. vor allem Verg. Aen. 6, 268-294.

${ }_{24}$ Sen. HF 813-816: postquam est ad oras Taenari ventum et nitor / percussit oculos lucis ignotae novus, I resumit animos victus et vastas furens / quassat catenas. 
dass Cerberus eine Zuflucht vor dem Sonnenlicht nur unter Hercules' Schatten finden kann, denn nur dort fühlt er sich wohl, ${ }^{25}$ entfaltet dabei eine transparente symbolische Bedeutung: Nicht nur bringt Hercules also das Infernalische im wörtlichen Sinne auf die Erde; er trägt es - für die Bewohner der Unterwelt bereits deutlich erkennbar — wie eine Metapher in sich.

Der metaphorische Status nicht nur des Übernatürlichen sondern der gesamten Handlung der Tragödie wird im dritten Chorlied mit besonderem Nachdruck betont. ${ }^{26}$ Nach seinem detaillierten Bericht über die Unterwelt wird Theseus plötzlich auf einen Festzug aufmerksam, dessen Mitglieder ein Lobeslied auf Hercules singen. ${ }^{27}$ Merkwürdigerweise fängt aber dieser angekündigte Lobesgesang erst ungefähr fünfzig Verse später an (der Übergang dazu wird durch einen Wechsel des Versmaßes deutlich gekennzeichnet). ${ }^{28}$ In diesem hinausgeschobenen zweiten Teil der Chorpassage haben wir es tatsächlich mit einem traditionell anmutenden Siegeshymnus zu tun. ${ }^{29}$ Diese festliche Freude steht allerdings in einem starken Kontrast zu den ersten zwei Dritteln der Chorpassage, die dem Hymnus vorangehen. Der Chor spricht hier zwar auch über die Katabasis des Hercules. Im Gegensatz zu Theseus' Schilderung der Unterwelt, werden hier keine mythologischen Monster beschrieben, sondern zunächst nur die unendliche Menge der Verstorbenen, die mit den Besuchern von öffentlichen Spektakeln - von einem Theaterspiel, von den Olympischen und den Pythischen Spielen und von den Eleusinischen Mysterien - verglichen werden. ${ }^{30}$ Verstorbene werden somit implizit als Zuschauer und die Unterwelt als Spektakel charakterisiert. Das Spektakel, das man hier zu Gesicht bekommt, besteht jedoch erstaunlicherweise nur aus Finsternis und Leere. ${ }^{31}$ Diese Erkenntnis wirkt nach dem mythologischen Bericht des Theseus, und im Kontext eines Lobesliedes anlässlich einer erfolgreichen Rückkehr aus der Unterwelt, ziemlich verstörend, besonders wenn der Chor explizit betont, dass niemand aus der Unterwelt zurückkehren kann. ${ }^{32}$ Das am Anfang der Passage gezeichnete Bild von Verstorbenen als Zuschauern im Unterwelttheater wird somit zum Bild von Theaterzuschauern - und das heißt, von uns als Rezipienten der Tragödie - als Sterbenden. Dass wir uns alle in dieser Passage angesprochen fühlen müssen, wird durch die allumfassende erste Person Plural in der abschließenden Sektion

${ }^{25}$ Sen. HF 821-827: vidit ut clarum diem / et pura nitidi spatia conspexit poli, / oborta nox est: lumina in terram dedit, / compressit oculos et diem invisum expulit / aciemque retro flexit atque omni petit / cervice terram; tum sub Herculeas caput / abscondit umbras.

${ }^{26}$ Kirichenko 2013, 260-263.

27 Sen. HF 827-829: densa sed laeto venit / clamore turba frontibus laurum gerens / magnique meritas Herculis laudes canit.

28 Fitch 1987, 334-335. Vgl. Davis 1993, 72-78.

29 Sen. HF 875ff.: Thebis laeta dies adest. / aras tangite supplices, / pingues caedite victimas; / permixtae maribus nurus / sollemnes agitent choros, etc.

${ }^{30}$ Sen. HF 838-849: quantus incedit populus per urbes / ad novi ludos avidus theatri, / quantus Eleum ruit ad Tonantem / quinta cum sacrum revocavit aetas; / quanta, cum longae redit hora nocti / crescere et somnos cupiens quietos / libra Phoebeos tenet aequa currus, / turba secretam Cererem frequentat / et citi tectis properant relictis / Attici noctem celebrare mystae: / tanta per campos agitur silentes / turba. Siehe Fitch 1987, 338-340.

31 Sen. HF 858-863: qualis est vobis animus, remota / luce cum maestus sibi quisque sensit / obrutum tota caput esse terra? / stat chaos densum tenebraeque turpes / et color noctis malus et silentis / otium mundi vacuaeque nubes.

32 Sen. HF 865-866: nemo ad id sero venit, unde numquam, / cum semel venit, poterit reverti. 
dieser Passage zusätzlich unterstrichen. ${ }^{33}$ Aus einem Theaterpublikum, das sich gerade noch von den visuellen Reizen der traditionellen Mythologie betören ließ, verwandeln wir uns in Zuschauer eines Spektakels, das aus nichts anderem als der absoluten Finsternis des Todes besteht - in dem auch in Senecas philosophischen Schriften unermüdlich betonten täglichen Sterben (cotidie mori) jedes einzelnen Menschen. ${ }^{34}$ Paradoxerweise wird aber dieses gestaltlose - bildlich undarstellbare - Spektakel der menschlichen Existenz dank dem Kontrast mit den darstellbaren, oder zumindest beschreibbaren, fiktionalen Bildern des Übernatürlichen überhaupt erst denkbar.

In Erfüllung des Versprechens, das Juno im Prolog gemacht hat, wird Hercules im vierten Akt wahnsinnig und stellt sich vor, dass er den Himmel mit Hilfe der aus der Unterwelt ausgebrochenen Giganten und Titanen erobert, die Kinder des Lycus tötet und sogar Juno selbst fängt. ${ }^{35}$ Es ist dabei besonders bezeichnend, dass sich das Geschehen gleichzeitig auf zwei Ebenen entfaltet: Während Hercules selbst seine wahnsinnigen Phantasien schildert, berichtet Amphitryon davon, wie Hercules seine Familie auf der Bühne tötet. ${ }^{36}$ Die Projektion der beiden Realitätsebenen - der übernatürlichen Wahnsinnsvision und der allzu menschlichen Bühnenhandlung - aufeinander erzeugt somit einen hybriden Raum, dessen Bestandteile nicht mehr getrennt wahrgenommen werden können. Die Grausamkeit der Tötung der eigenen Familie wird einerseits in der mythologischen Begrifflichkeit der archetypischen Verbrechen der Titanen und der Giganten verstanden - und indirekt auf das gleiche kosmische Niveau gehoben. Andererseits lässt die visuelle Unmittelbarkeit der durch den Bühneraum fliegenden Körperteile und der aus dem Kopf eines Kindes herausfließenden Gehirnmasse, ${ }^{37}$ die literarische Klischeehaftigkeit dieses kosmischen Konflikts geradezu harmlos erscheinen, denn das, was wir auf der Bühne sehen, erweist sich in der Tat als schlimmer denn die schlimmsten literarischen Schreckensbilder. Zu dieser Erkenntnis kann man aber offensichtlich nur anhand des auf der Bühne konstruierten hybriden Raumes kommen, in dem das visuell Wahrnehmbare durch übernatürliche literarische Gestalten überlagert wird.

Die mythologisch übernatürliche Bildlichkeit erweist sich im vierten - und letzten - Auftritt des Chors als geradezu unentbehrlich für die Schilderung emotional ver-

${ }^{33}$ Sen. HF 872-874: parce venturis: tibi, mors, paramur; / sis licet segnis, properamus ipsi: / prima quae vitam dedit hora carpit.

${ }^{34}$ Zur zentralen Bedeutung der Vorstellung vom Leben als einem graduellen Tod (cotidie mori) für Senecas philosophisches Denken siehe Schönegg 1999, 95-98; Ker 2009, 147-176. Vgl. Sen. ep. 1, 2: quem mihi dabis qui aliquod pretium tempori ponat, quo diem aestimet, qui intellegat se cotidie mori? 24, 20: cotidie morimur; cotidie enim demitur aliqua pars vitae, et tunc quoque cum crescimus vita decrescit. Vgl. auch Sen. ep. 58, 23.

${ }^{35}$ Sen. HF 955-973 (Eroberung des Himmels), 967-981 (die Giganten und die Titanen), 987-989 (die Kinder des Lycus), 1018 (teneo novercam). Die Tatsache, dass der senecanische Hercules in seinem Wahnsinn meint, er töte Lycus' Kinder, und nicht, wie sein Vorgänger bei Euripides, diejenigen des Eurystheus (Eur. Her. 963-989), dient dazu, die Verbindung zwischen den beiden Figuren noch weiter zu vertiefen. Vgl. Littlewood 2004, 25-36. Einen ausführlichen Vergleich zwischen den Darstellungen des Wahnsinns des Hercules bei Euripides und bei Seneca findet man in Papadopoulou 2004. Zu den Symptomen des Wahnsinns in Senecas HF siehe Auvray 1989, 85-90. Zu ihren kosmischen Ausmaßen siehe Schmitz 1993, 127-134.

${ }^{36}$ Vgl. Wessels 2014, 232-234.

37 Sen. HF 1004-1007: scelus nefandum, triste et aspectu horridum: / dextra precantem rapuit et circa furens / bis ter rotatum misit; ast illi caput / sonuit, cerebro tecta disperso madent; 1024-1026 in coniugem nunc clava libratur gravis: / perfregit ossa, corpori trunco caput / abest nec usquam est. Vgl. Wessels 2014, 245-249. 
störender Ereignisse: Ungeachtet seiner bisherigen Skepsis gegenüber mythologischen Vorstellungen singt nun der Chor ein Klagelied für die ermordete Familie des Hercules, in dem der grenzenlose Schmerz, den der Mörder selbst nach seinem Aufwachen verspüren wird, ausschließlich in der Begrifflichkeit der kosmischen Mythologie aufgefasst wird. ${ }^{38}$ Als Hercules dann am Anfang des fünften Aktes aufwacht und sich von den zerstückelten Körpern seiner Ehefrau und seiner Söhne sieht, sieht er gleichzeitig den hybriden Raum, den er selbst erschaffen hat, denn er meint sofort, „sein Geist sei die Gestalten der Unterwelt noch nicht losgeworden. ${ }^{\text {" }} 9$ Die echte Unterwelt erlebt Hercules also, wie es Juno im Prolog betonte, ${ }^{40}$ nicht in einem fantastisch mythischen Bereich, sondern hier - in der empirischen Banalität der Bühnenwelt. Als er endgültig begreift, was geschehen ist, besteht seine unmittelbare Reaktion darauf in dem Wunsch, diesen hybriden Raum in seine ursprünglichen Bestandteile zu zerlegen — das heißt, sein unsägliches Verbrechen durch den Selbstmord zu bestrafen, den er als Verbannung in den tiefsten, weder dem Cerberus noch ihm selbst bekannten Winkel der Unterwelt konzipiert. ${ }^{41}$ Mehr noch: wie von Juno im Prolog bereits angedeutet, versteht er nun seinen Tod als eine Art überbietende Nachahmung des mythologischen Musters, das sein Leben bis jetzt definierte - nämlich als seine größte Heldentat: Seine Heldentaten bestanden bekanntlich vor allem in der Tötung von Monstern, und er ist im Moment nun mal das schlimmste Monster, das es gibt. ${ }^{42}$ Sein Selbstmordwunsch lässt sich somit als Ausdruck seines Wunsches deuten, wieder zu einer monolithischen Heldengestalt zu werden, die er einmal war, und dabei einen klar definierten, unvermischten mythologischen Raum zu bewohnen - und sei es diesmal der Raum der Unterwelt. So etwas erweist sich jedoch als vollkommen unmöglich: Es stellt sich nämlich heraus, dass die Fortsetzung des bewahrten mythologischen Musters keine feinsäuberliche Aufspaltung des hybridisierten Raumes und keine Rückkehr zum unvermischten, unschuldigen Heroismus mit sich bringen würde, sondern nur noch ein weiteres Verbrechen, das auf Hercules lasten würde: Amphitryon lässt keinen Zweifel daran übrig, dass er sich nach Hercules' Tod auch umbringen und seinen Sohn dafür verantwortlich machen würde. ${ }^{43}$ Hercules' Entschluss, am Leben zu bleiben, vollendet dann die Hybridisierung des Raums, die sich im Verlaufe der Tragödie Schritt für Schritt vollzog. Hercules' Mord an seiner Familie verwandelte die empirisch mögliche Bühnenwelt in eine Projektion der fantastischen Domäne der Unterwelt. Wenn aber Hercules am Ende der Tragödie sagt „Auch diese Tat zähle zu den Herkules-Taten: ich soll leben, “44 verwandelt

38 Sen. HF 1104-1114: gemitus vastos audiat aether, / audiat atri regina poli / vastisque ferox qui colla gerit / vincta catenis / imo latitans Cerberus antro. / resonet maesto clamore chaos / latique patens unda profundi / et qui medius tua tela tamen / senserat aer. / pectora tantis obsessa malis / non sunt ictu ferienda levi: / uno planctu tria regna sonent.

39 Sen. HF 1143-1146: unde prostrata ad domum / video cruenta corpora? an nondum exuit / simulacra mens inferna? post reditus quoque / oberrat oculis turba feralis meis?

40 Sen. HF 91: hic tibi ostendam inferos.

41 Sen. HF 1221-1226: dira Furiarum loca / et inferorum carcer et sonti plaga / decreta turbae - si quod exilium patet / ulterius Erebo, Cerbero ignotum et mihi, / hoc me abde, Tellus; Tartari ad finem ultimum Imansurus ibo. Billerbeck 2014, 429-430.

42 Sen. HF 1279-1282: purgare terras propero. iamdudum mihi / monstrum impium saevumque et immite ac ferum / oberrat: agedum, dextra, conare aggredi / ingens opus, labore bis seno amplius.

43 Claudia Wiener (2006, 98-102) wertet diese Interaktion als „ein erfolgreiches Therapiegespräch.“ Zu Hercules' Ablehnung des Selbstmordes am Ende der Tragödie siehe auch Palmieri 1999, 132-149; Wessels $2014,106-110$.

44 Sen. HF 1316-1317: eat ad labores hic quoque Herculeus labor: / vivamus. 
er die Banalität des menschlichen Lebens, die der Chor in seinem ersten Lied so überschwänglich lobte, in eine Projektion der heroischen Bühnenhandlung: Als die größte Heldentat des Hercules galt bis dahin seine Katabasis; ${ }^{45}$ nun kommt etwas so Banales wie das Leben als solches (vivamus) hinzu - eine Heldentat, die nach der im Laufe des Stücks konsequent angewendeten heroischen Logik, größer sein muss als die Bezwingung der Monstern der Unterwelt. ${ }^{46}$ Hercules' Entscheidung, die verwirrende Komplexität des Lebens zu konfrontieren, anstatt in der sicheren, feinsäuberlich kategorisierten Welt der mythologischen Fiktion Zuflucht zu suchen, wird somit zu einer Parabel für das menschliche Leben als solches, das unter Umständen zu einer Heldentat werden kann. ${ }^{47}$

Dem scheinbar banalen Gedanken, am Leben zu bleiben sei unter Umständen heldenhafter als zu sterben, begegnen wir zwar auch in Senecas philosophischen Schriften. ${ }^{48}$ Es ist jedoch die innerhalb des imaginären Bühnenraums der senecanischen Tragödie stattfindende Vermischung zwischen dem Mythologischen und dem Alltäglichen, die dazu beiträgt, dass dieser Gedanke eine emotional besonders wirksame Überzeugungskraft entfaltet. Das in der Tragödie inszenierte introspektive Sehen des Unsichtbaren scheint somit einen im Grunde philosophischen - protreptischen - Zweck zu verfolgen. Denn durch diese schmerzhafte Visualisierung wird schließlich jeder Rezipient der Tragödie indirekt dazu animiert, mit dem mühsamen Prozess seiner eigenen philosophischen Selbsttherapie zu beginnen. ${ }^{49}$

\section{Literaturhinweise}

Auvray C.-E. Folie et douleur dans Hercule furieux et Hercule sur l'Oeta: Recherches sur l'expression esthétique de l'ascèse stoïcienne chez Sénèque. Frankfurt 1989.

Aygon J.-P. Pictor in fabula: l’ecphrasis - descriptio dans les tragédies de Sénèque. Brüssel 2004.

Bartsch S. The Mirror of the Self: Sexuality, Self-Knowledge, and the Gaze in the Early Roman Empire. Chicago 2006. wird.

${ }^{45}$ Vgl. Sen. HF 529-557, wo die Katabasis als die letzte — und somit die größte - Heldentat erwähnt

${ }^{46}$ Es darf wohl kaum überraschen, dass man in Euripides' Herakles nichts Vergleichbares findet. Bei Euripides wird Herakles zwar auch - dieses Mal von Theseus - erfolgreich überredet, von einem Selbstmord abzusehen; dies geschieht jedoch mit der Begründung, er könne sich in einem der zahlreichen athenischen Heiligtümer einer rituellen Reinigung unterziehen: Eur. Her. 1313-1420; vgl. die letzten Worte von Senecas Hercules Furens (Sen. HF. 1341-1344): nostra te tellus manet. / illic solutam caede Gradivus manum / restituit armis: illa te, Alcide, vocat, / facere innocentes terra quae superos solet. Zum Vergleich zwischen den Schlusspartien der beiden Tragödien siehe Lieberg 1994.

47 Kirichenko 2013, 27-33.

48 Sen. ep. 78, 2: saepe impetum cepi abrumpendae vitae: patris me indulgentissimi senectus retinuit. cogitavi enim non quam fortiter ego mori possem, sed quam ille fortiter desiderare non posset. itaque imperavi mihi, ut viverem. aliquando enim et vivere fortiter facere est. 104, 3 indulgendum est enim honestis adfectibus; et interdum, etiam si premunt causae, spiritus in honorem suorum vel cum tormento revocandus et in ipso ore retinendus est, cum bono viro vivendum sit non quamdiu iuvat sed quamdiu oportet: ille qui non uxorem, non amicum tanti putat ut diutius in vita commoretur, qui perseverabit mori, delicatus est. hoc quoque imperet sibi animus, ubi utilitas suorum exigit, nec tantum si vult mori.

${ }^{49}$ Für eine detaillierte Besprechung des protreptischen Potenzials der senecanischen Tragödien (in Verbindung mit seinen philosophischen Schriften) siehe Kirichenko (2013), 207-279. Vgl. Epict. 3, 23,

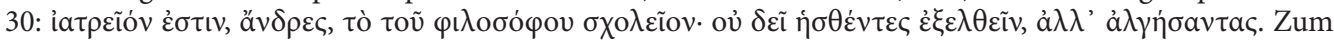
antiken Topos der Bekehrung zur Philosophie siehe Kirichenko (2010), 87-105. Zur Therapie in Senecas Briefen siehe Graver (1996) und Dietsche (2014); in De ira, Nussbaum (1994), 402-438; im Stoizismus im Allgemeinen, Sorabji (2000), 159-300. Vgl. Sen. ep. 6, 1 et hoc ipsum argumentum est in melius translati animi, quod vitia sua, quae adhuc ignorabat, videt; Bartsch (2006), 191-229. 
Bartsch S. - Schiesaro, A. (Hgg.) The Cambridge Companion to Seneca. Cambridge 2015.

Billerbeck M. (Hg.) Seneca. Hercules furens: Einleitung, Text, Übersetzung und Kommentar. Leiden 1999.

Billerbeck M. Hercules Furens. In: Damschen - Heil 2014, 425-434.

Chaumartin F.-R. Philosophical Tragedy? In: Damschen - Heil 2014, 653-669.

Damschen G. - Heil, A. (Hgg.) Brill's Companion to Seneca: Philosopher and Dramatist. Leiden 2014.

Davis P. J. Shifting Song: The Chorus in Seneca' Tragedies. Hildesheim 1993.

Degl'Innocenti Pierini, R. Venit ad pigros cana senectus (Sen. Herc. F. 198). Un motivo dei cori senecani tra filosofia ed attualità, in: Castagna, L.(Hg.) Nove studi sui cori tragici di Seneca. Mailand 1996, 37-56.

Dietsche U. Strategie und Philosophie bei Seneca: Untersuchungen zur therpeutischen Technik in den Epistulae morales. Berlin 2014.

Dingel J. Seneca und die Dichtung. Heidelberg 1974.

Fantham E. Seneca's Troades. A Literary Introduction with Text, Translation, and Commentary. Princeton 1982.

Fauconier, G. - Turner, M. The Way We Think: Conceptual Blending and the Mind's Hidden Complexities. New York 2002.

Fischer S. Seneca als Theologe. Berlin 2008.

Fitch J. G. (Hg.) Seneca: Hercules furens. Edited with introduction and commentary. Ithaca, NY 1987.

Gärtner Th. 'Besser dem gemeinen Volk anzugehören': Zur Rolle des Chors in der senecanischen Tragödie. Studia Humaniora Tartuensia, 2003, 4. A. 4, 1-53.

Graver M.E. Therapeutic Reading and Seneca's Moral Epistles. Providence (Diss., Brown University), 1996.

Guastella G. L'ira e lonore: forme della vendetta nel teatro senecano e nella sua tradizione. Palermo 2001.

Harrison G. W. (Hg.) Seneca in Performance. Swansea 2000.

Heil A. Die dramatische Zeit in Senecas Tragödien. Leiden 2013.

Ker J. The Deaths of Seneca. Oxford 2009.

Kirichenko A.A Comedy of Storytelling: Theatricality and Narrative in Apuleius' Golden Ass. Heidelberg 2010.

Kirichenko A.Lehrreiche Trugbilder: Senecas Tragödien und die Rhetorik des Sehens. Heidelberg 2013.

Kohn Th. The Dramaturgy of Senecan Tragedy. Ann Arbor 2013.

Kugelmeier Chr. Die innere Vergegenwärtigung des Bühnenspiels in Senecas Tragödien. München 2007.

Lawall G. Virtus and pietas in Seneca's Hercules Furens. Ramus 1983, 12, 6-26.

Lefèvre E. Die philosophische Bedeutung der Seneca-Tragödie am Beispiel des Thyestes, in: W. Haase (Hg.), Aufstieg und Niedergang der römischen Welt, 2, 32, 2, Berlin 1985, 1263-1283.

Li Causi P. Nella rete di Giunone: cause, forme e finalità della vendetta nell'Hercules Furens di Seneca. Dioniso 2006, 5, 118-137.

Lieberg G. Gli atti finali dell'Eracle di Euripide e dell'Hercules furens di Seneca, in: Curti, C. - Crimi C. (Hg.) Scritti classici e cristiani offerti a Francesco Corsaro. Catania 1994, 385-413.

Littlewood C. Self-Representation and Illusion in Senecan Tragedy. Oxford 2004.

Mader G. Form and Meaning in Seneca's Dawn Song (H. F. 125-201). AClass 1990, 33, 1-32.

Marti B. Seneca’s Tragedies. A New Interpretation. TAPhA 1945, 76, 216-245.

Nisbet R. G. M. The Dating of Seneca's Tragedies, with Special Reference to Thyestes. ARCA 1990, 29, 95-114.

Nussbaum M. Poetry and the Passions: Two Stoic Views. In: Brunschwig, J. - Nussbaum M. C. (Hg.) Passions and Perceptions: Studies in Hellenistic Philosophy of Mind. Cambridge 1993, 97-149.

Nussbaum M.C. The Therapy of Desire: Theory and Practice in Hellenistic Ethics. Princeton 1994.

Palmieri N. L'eroe al bivio: modelli di „mors voluntaria“ in Seneca tragico. Pisa 1999.

Papadopoulou Th. Herakles and Hercules: The Hero's Ambivalence in Euripides and Seneca. Mnemosyne 2004, 57, 257-283.

Pratt N. Seneca's Drama. Chapel Hill 1983.

Regenbogen O.Schmerz und Tod in den Tragödien Senecas. Darmstadt 1963 (= Vorträge der Bibliothek Warburg 1927/1928, 7).

Rosenmeyer Th. G. Senecan Drama and Stoic Cosmology. Berkeley 1989.

Schiesaro A. The Passions in Play: Thyestes and the Dynamics of Senecan Drama. Cambridge 2003.

Schmitz Chr. Die kosmische Dimension in den Tragödien Senecas. Berlin 1993.

Schönegg B. Senecas epistulae morales als philosophisches Kunstwerk. Bern 1999.

Segal Ch. Language and Desire in Seneca's Phaedra. Princeton 1986.

Shelton J. A. Seneca's Hercules Furens: Theme, Structure and Style. Göttingen 1978.

Solimano G. La prepotenza dellocchio: riflessioni sullopera di Seneca. Genova 1991. 
Sorabji R. Emotions and Peace of Mind: From Stoic Agitation to Christian Temptation. Oxford 2000.

Staley G. A. Seneca and the Idea of Tragedy. Oxford 2010.

Sutton D. F. Seneca on the Stage. Leiden 1986.

Tarrant R. J. Seneca. Agamemnon. Edited with a Commentary. Cambridge 1976.

Tarrant R. J. Senecan Drama and its Antecedents. HSCP 1978, 82, 213-263.

Trinacty Ch. Senecan Tragedy and the Reception of Augustan Poetry. Oxford 2014.

Trinacty Ch. Senecan Tragedy. In: Bartsch - Schiesaro 2015, 29-40.

Turner M. The Literary Mind. Oxford 1996.

Wessels A. Ästhetisierung und ästhetische Erfahrung von Gewalt: Eine Untersuchung zu Senecas Tragödien. Heidelberg 2014.

Wiener C. Stoische Doktrin in römischer Belletristik: das Problem von Entscheidungsfreiheit und Determinismus in Senecas Tragödien und Lucans Pharsalia. München 2006.

Winter K. Artificia mali: Das Böse als Kunstwerk in Senecas Rachetragödien. Heidelberg 2014.

Zwierlein O.Die Rezitationsdramen Senecas. Meisenheim am Glan 1966.

For citation: Kirichenko A. Der imaginäre Bühnenraum in Senecas Hercules Furens. Philologia Classica 2016, 11(2), 258-268. DOI: 10.21638/11701/spbu20.2016.205

\section{ВООБРАЖАЕМОЕ СЦЕНИЧЕСКОЕ ПРОСТРАНСТВО В ТРАГЕДИИ СЕНЕКИ «ГЕРКУЛЕС В БЕЗУМЬЕ»}

\section{Александр Леонидович Кириченко}

Целью данной статьи является анализ построения пространства в трагедии Сенеки Безумный Геркулес. Статья демонстрирует, что трагедия базируется на структурной аналогии между тремя концептуальными областями - 1) миром сверхъестественной мифологии, который не может быть правдоподобно показан на сцене и поэтому детально описывается в дескриптивных пассажах, 2) визуально воспринимаемым миром сценического действия и 3) абстрактно мыслимым миром 'человеческой жизни'. Эти три области сливаются в единое воображаемое пространство, в рамках которого философская мысль оказывается не столько сформулированной, сколько наглядно инсценированной, подвергая зрителя /читателя эмоциональному воздействию, потенциально способствующему его внутренней трансформации. Таким образом, помимо интерпретации трагедии Безумный Геркулес данная статья предлагает новый общий подход к роли философии в драмах Сенеки.

Ключевые слова: Сенека, Безумный Геркулес, трагедия, философия. 